\title{
Characterising Charm Jet Properties with Azimuthal Correlations of D Mesons and Charged Particles with ALICE at the $\mathrm{LHC}^{+}$
}

\author{
Shyam Kumar (1) for the ALICE Collaboration \\ Indian Institute of Technology, Bombay, Mumbai 400076, India; shyam.kumar@cern.ch; Tel.: +91-7208246534 \\ + Presented at Hot Quarks 2018-Workshop for Young Scientists on the Physics of Ultrarelativistic \\ Nucleus-Nucleus Collisions, Texel, The Netherlands, 7-14 September 2018.
}

Published: 19 April 2019

\begin{abstract}
Charm quarks are produced via hard parton scattering in ultra-relativistic heavy-ion collisions, hence are ideal probes to study a possible de-confined state of matter, known as Quark Gluon Plasma (QGP). The angular correlation of a meson containing a charm quark with other charged particles in heavy-ion collisions can help in studying the properties of QGP. Similar studies in $\mathrm{pp}$ collisions can give insight about the charm production mechanism while in $\mathrm{p}-\mathrm{Pb}$ collisions could provide essential information to disentangle final-state QGP-induced modifications from effects caused by cold nuclear matter. In this proceedings, the results are presented for $\mathrm{p}-\mathrm{Pb}$ collisions at $\sqrt{s_{\mathrm{NN}}}=5.02 \mathrm{TeV}$ and pp collisions at $\sqrt{s}=13 \mathrm{TeV}$, so far the highest available energy at the LHC. The results are compared with Monte Carlo (MC) simulations using PYTHIA and POWHEG event generators and with pp collision results at $\sqrt{\mathrm{s}}=7 \mathrm{TeV}$.
\end{abstract}

Keywords: charm production mechanism; cold nuclear matter effect; Quark Gluon Plasma

\section{Introduction}

The azimuthal correlation of a D-meson with charged particles in pp collisions can give insight into the charm production mechanisms. There are three main charm production mechanisms: pair production, flavor excitation, and gluon splitting [1]. The pair production is a leading order [LO] process while flavor excitation and gluon splitting are next to leading order [NLO] processes as shown in Figure 1. The correlation of D-meson in pp collisions as shown in the center of Figure 2 can constrain the perturbative QCD inspired Monte-Carlo (MC) models, as well as serve as a reference for $\mathrm{p}-\mathrm{Pb}$ and $\mathrm{Pb}-\mathrm{Pb}$ collisions. In $\mathrm{p}-\mathrm{Pb}$ collisions, we have initial state effects like shadowing, $k_{\mathrm{T}}$ broadening (Cronin effect), gluon saturation, and energy loss in cold nuclear matter while in $\mathrm{Pb}-\mathrm{Pb}$ collisions we have final state effects due to the medium formation together with initial state effects [2,3]. The study of azimuthal correlation in $\mathrm{p}-\mathrm{Pb}$ collisions is necessary to disentangle final state effects (energy loss, fragmentation, and recombination) from the initial state effects. In addition, it also allows searching for long-range double-ridge structure in the heavy-flavor sector as shown in Figure 2 [3]. ALICE has performed measurements of D-hadron correlation in pp collisions and $\mathrm{p}-\mathrm{Pb}$ collisions at $\sqrt{s}=13 \mathrm{TeV}$ and $\sqrt{s_{\mathrm{NN}}}=5.02 \mathrm{TeV}$, respectively with ALICE at the LHC. We have compared the near-side (NS) and away-side (AS) jet properties for $\mathrm{pp}$ and $\mathrm{p}-\mathrm{Pb}$ collisions and also compared the data with perturbative QCD inspired MC models, PYTHIA [LO] [4,5] and POWHEG [NLO] [6]. The study of the correlation in pp collisions at $\sqrt{s_{\mathrm{NN}}}=5.02 \mathrm{TeV}$ is ongoing and it will be of primary importance to assess possible modifications of the correlation peak due to the presence of cold nuclear matter effects. 


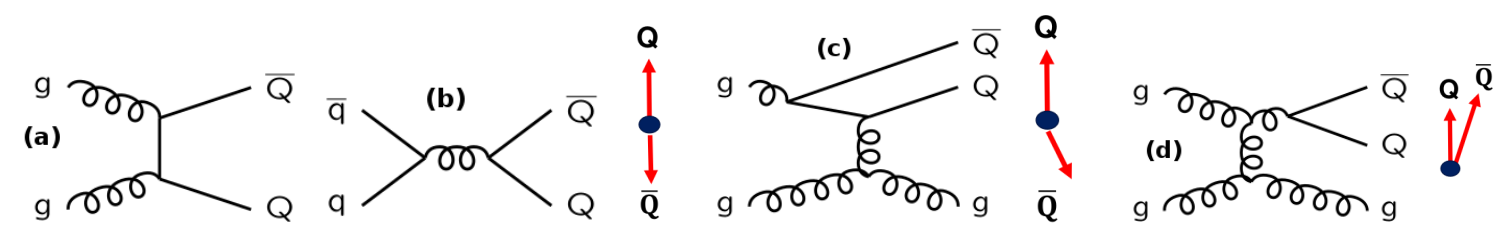

Figure 1. Charm production mechanisms: (a) gluon fusion, (b) quark annihilation, in the pair production (LO), the heavy quarks are back to back at $180^{\circ}$, (c) flavor excitation (NLO), the heavy quarks have a large opening angle, (d) gluon splitting (NLO), the heavy quarks have a very small opening angle [1].
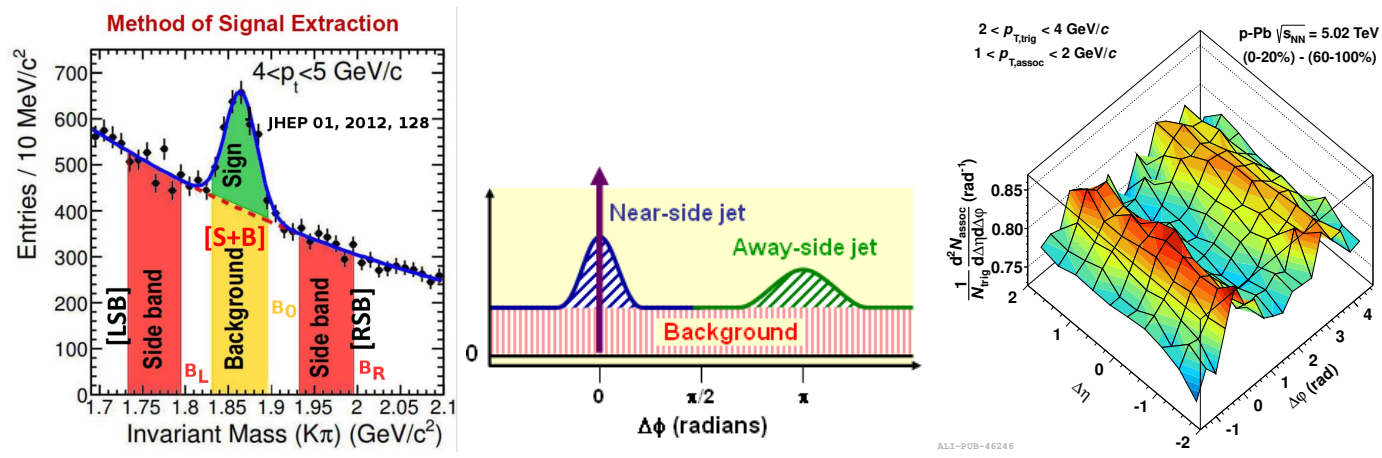

Figure 2. (Left) Side-band subtraction from invariant mass distributions, (Center): near side, away side peak and baseline [7], (Right) The double ridge structure observed in di-hadron correlations [3].

\section{Analysis Details}

The study is performed on pp 2017 and p-Pb 2016 Run 2 data at $\sqrt{s}=13 \mathrm{TeV}$ and $\sqrt{s_{\mathrm{NN}}}=5.02 \mathrm{TeV}$, respectively, collected by the ALICE detector at the LHC [8]. In the analysis, we used following sub-detectors of ALICE, the inner tracking system (ITS) for tracking and reconstruction of primary and secondary vertices, time projection chamber (TPC) for tracking and particle identification, and time of flight detector (TOF) for particle identification. The D-meson $\left(\mathrm{D}^{+}, \mathrm{D}^{0}\right.$, and $\left.\mathrm{D}^{*+}\right)$ reconstruction from its daughters as given in Table 1 is done using topological cuts and particle identification information to reduce the combinatorial background. An invariant mass analysis is performed in order to separate the signal (Gaussian fit) and background (exponential fit) as shown in the left panel of Figure 2. $\mathrm{D}$ meson candidates are correlated with primary charged particles $(\mathrm{e}, \mu, \pi, \mathrm{K}$, and $\mathrm{p})$ by calculating $\Delta \eta=\eta_{\text {trigger }}-\eta_{\text {assoc }}$ and $\Delta \phi=\phi_{\text {trigger }}-\phi_{\text {assoc }}$ in the same event. The correlation of signal D-meson is extracted by using the method of side-band subtraction as given by the expression below.

$$
\text { Signal correlation }=\text { Correlation in }[S+B] \text { region }-[\text { Correlation in }[\mathrm{LSB}+\mathrm{RSB}] \text { region }] \times \mathrm{SF}
$$

where signal correlation is the correlation of the true D-meson with the charged particles. SF is the scale factor calculated from the ratio of the yield in the background region and side-band (left LSB, right RSB) region and is given by $B_{0} /\left(B_{L}+B_{R}\right)$. The distribution is corrected for the detector acceptance and inhomogeneity by using mixed-event techniques, in which we mix the event with the same z-vertex and multiplicity. The majority of secondary particles is removed by using distance of closest approach cuts, $\mathrm{DCA}_{\mathrm{xy}}$ and $\mathrm{DCA}_{\mathrm{z}}$. The distribution is also corrected for secondary track contaminations from strange decays and conversion inside the detector material by evaluating the fraction of accepted secondary tracks from MC simulation. The distribution is further corrected for the D-meson reconstruction efficiency, tracking efficiency, bias induced due to B-hadron to D-hadron decay topology. The final correction is the subtraction of contribution from the D-meson coming from the B-hadron decay. Finally, we study the per trigger normalized correlation distributions of D-mesons with primary charged particles. These fully corrected correlation distributions are projected on $\Delta \phi$ axis for the interval $|\Delta \eta|<0.9$. Next, the weighted average of all three D-mesons is done. In order to 
reduce the statistical uncertainty the reflection from $\left[-\frac{\pi}{2}, \frac{3 \pi}{2}\right]$ to $[0, \pi]$ is done as shown in the left panel of Figure 3. Finally, we fit the $\Delta \phi$ distribution by the following equation:

$$
f(\Delta \phi)=c+\frac{Y_{N S}}{\sqrt{2 \pi} \sigma_{N S}} e^{\frac{-\left(\Delta \phi-\mu_{N S}\right)^{2}}{2 \sigma_{N S}^{2}}}+\frac{Y_{A S}}{\sqrt{2 \pi} \sigma_{A S}} e^{\frac{-\left(\Delta \phi-\mu_{A S}\right)^{2}}{2 \sigma_{A S}^{2}}}
$$

Equation (2), which include the near-side (NS) peak, away-side (AS) peak, a baseline and together with a double Gaussian [9].

Table 1. D-mesons decay mode and their branching ratios [10].

\begin{tabular}{cc}
\hline D-Meson Decay Mode & Branching Ratios \\
\hline$D^{+} \rightarrow K^{-} \pi^{+} \pi^{+}$ & $9.13 \pm 0.19 \%$ \\
$D^{0} \rightarrow K^{-} \pi^{+}$ & $3.88 \pm 0.05 \%$ \\
$D^{*+} \rightarrow D^{0} \pi^{+}$ & $67.7 \pm 0.50 \%$ \\
\hline
\end{tabular}
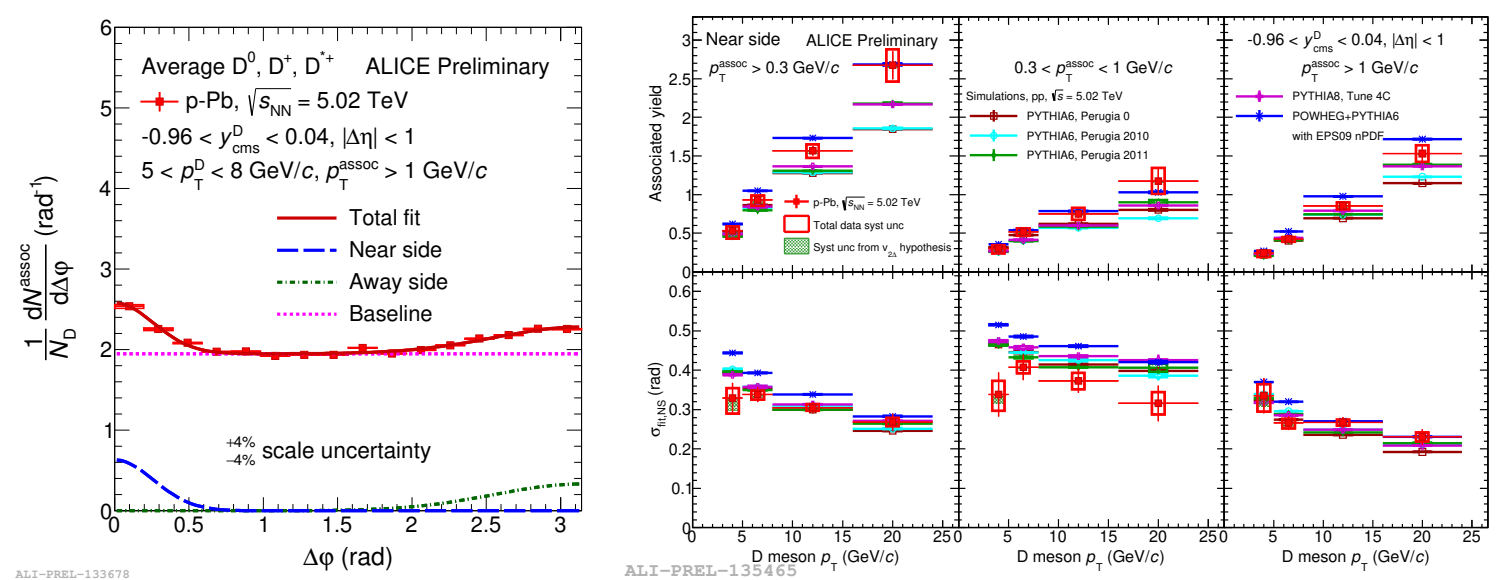

Figure 3. (Left) Example of reflected correlation distribution fitted with a double Gaussian and a constant baseline and (Right) comparison of NS yield and Gaussian width $\left(\sigma_{\mathrm{NS}}\right)$ with the MC models.

\section{Results}

The D-meson trigger particles are selected in different $p_{\mathrm{T}}$ ranges $(3-5,5-8,8-16$, and $16-24 \mathrm{GeV} / c)$ and correlated with the associated charged particles in three $p_{\mathrm{T}}$ bins $\left(p_{\mathrm{T}}^{\text {assoc }}>0.3 \mathrm{GeV} / c\right.$, $p_{\mathrm{T}}^{\text {assoc }}=0.3-1.0 \mathrm{GeV} / c$, and $\left.p_{\mathrm{T}}^{\text {assoc }}>1.0 \mathrm{GeV} / c\right)$. An exemplary plot for the D-meson $p_{\mathrm{T}}=5-8 \mathrm{GeV} / c$ and $p_{\mathrm{T}}^{\text {assoc }}>1.0 \mathrm{GeV} / c$ clearly demonstrating the NS and AS peaks after the baseline subtraction in the left panel of Figure 3 by blue and green dashed lines, respectively. These peaks characterize the properties of associated tracks in the jet created by a charm quark. The integration of blue dashed line over $\Delta \varphi$ gives the number of the associated particles of $p_{\mathrm{T}}>1.0 \mathrm{GeV} / c$ in the NS-jet for a trigger D-meson in the $p_{\mathrm{T}}$ range of 5-8 GeV/c. The results for the NS-peak shown in the right panel of Figure 3 imply that the yield increases with decreasing $\sigma$ of the peak as the $p_{\mathrm{T}}$ of D-meson increases. The results can be interpreted as number of associated particles in the NS jet increases along with the NS jet collimation. In Figure 4, the NS yield and width are compared for p-Pb data $\left(\sqrt{s_{N N}}=5.02 \mathrm{TeV}\right)$ with pp data at $\sqrt{s}=7,13 \mathrm{TeV}$. The comparison shows that the results are compatible within the uncertainties. 


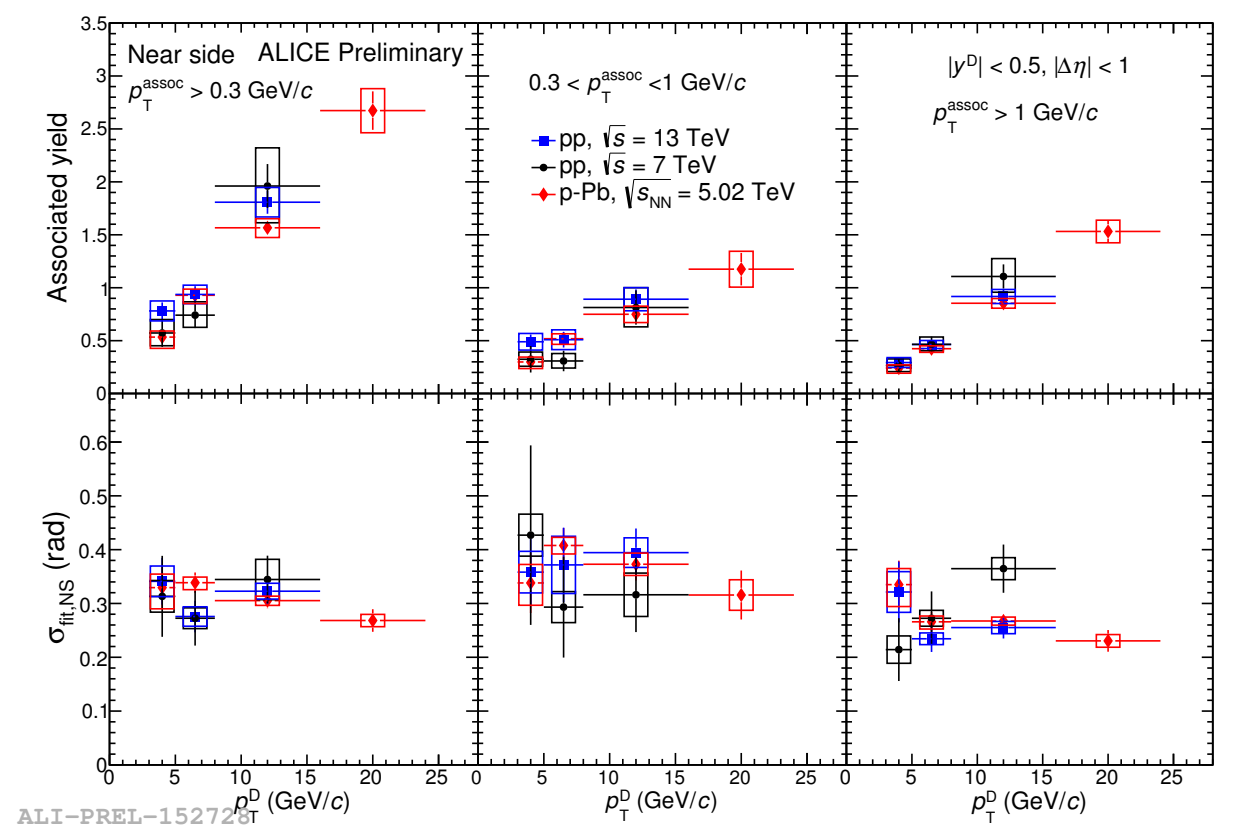

Figure 4. Comparison of NS yield and Gaussian width $\left(\sigma_{\mathrm{NS}}\right)$ for pp data at $\sqrt{s}=7,13 \mathrm{TeV}$ and $\mathrm{p}-\mathrm{Pb}$ data at $\sqrt{s_{\mathrm{NN}}}=5.02 \mathrm{TeV}$.

\section{Conclusions}

In $\mathrm{p}-\mathrm{Pb}$ collisions, the yield of associated charged particles increases with the $p_{\mathrm{T}}$ of $\mathrm{D}$-mesons while the Gaussian width of the near-side peak decreases. The comparison of $\mathrm{p}-\mathrm{Pb}$ data with $\mathrm{pQCD}$ inspired MC models like PYTHIA [LO] and POWHEG [NLO] shows that the data is compatible with models within uncertainties. Finally, the measurement in $\mathrm{p}-\mathrm{Pb}$ collisions is also compared with that in pp collisions and within uncertainties both measurements are consistent.

Acknowledgments: I want to thank the ALICE utilization and upgrade project, Department of Science and Technology (DST), India for the research and travel support.

\section{References}

1. Norrbin, E.; Sjostrand, T. Production and hadronization of heavy quarks. Eur. Phys. J. C 2000, 17, 137-161, doi:10.1007/s100520000460.

2. Liu, F.M.; Liu, S.X. Quark-gluon plasma formation time and direct photons from heavy ion collisions. Phys. Rev. C 2014, 89, 034906, doi:10.1103/PhysRevC.89.034906.

3. Kamin, J. Hot and cold nuclear matter effects in $\mathrm{p}-\mathrm{Pb}$ collisions at the LHC. In Proceedings of the 3rd International Conference on New Frontiers in Physics, Crete, Greece, 28 July-06 August 2014; Volume 95, doi:10.1051/epjconf/20159503018.

4. Sjostrand, T.; Mrenna, S.; Skands, P.Z. PYTHIA 6.4 Physics and Manual. JHEP 2006, 2006, doi:10.1088/1126-6708/2006/05/026.

5. Sjostrand, T.; Mrenna, S.; Skands, P.Z. A Brief Introduction to PYTHIA 8.1. Comput. Phys. Commun. 2008, 178, 852-867, doi:10.1016/j.cpc.2008.01.036.

6. Frixione, S.; Nason, P.; Oleari, C. Matching NLO QCD computations with Parton Shower simulations: The POWHEG method. JHEP 2007, 2007, doi:10.1088/1126-6708/2007/11/070.

7. BNL. Star Collaboration star focus, "jets in nuclear collisions". In The Star Experiment at RHIC; BNL: Rome, Italy, 2006. Available online: https://www.star.bnl.gov/central/focus/highPt/ (acessed on 12 April 2019)

8. Aamodt, K.; Quintana, A.A.; Achenbach, R.; Acounis, S.; Adamová, D.; Adler, C.; Aggarwal, M.; Agnese, F.; Rinella, G.A. The ALICE experiment at the CERN LHC. JINST 2008, 3, S08002, doi:10.1088/1748-0221/3/08/S08002. 
9. Adam, J.; Bregant, M.; Munhoz, M.G.; Suaide, A.A.d.P. Measurement of azimuthal correlations of D mesons and charged particles in pp collisions at $\sqrt{s}=7 \mathrm{TeV}$ and p-Pb collisions at $\sqrt{s_{\mathrm{NN}}}=5.02$ TeV. Eur. Phys. J. C 2017, 77, 245, doi:10.1140/epjc/s10052-017-4779-8.

10. Patrignani, C.; Agashe, K.; Aielli, G.; Amsler, C.; Antonelli, M.; Asner, D.M.; Baer, H.; Banerjee, S.; Barnett, R.M.; Basaglia, T. Review of Particle Physics. Chin. Phys. C 2016, 40, 100001, doi:10.1088/1674-1137/40/10/100001.

(C) 2019 by the authors. Licensee MDPI, Basel, Switzerland. This article is an open access article distributed under the terms and conditions of the Creative Commons Attribution (CC BY) license (http:/ / creativecommons.org/licenses/by/4.0/). 